\title{
Profil de la polyarthrite rhumatoïde en consultation rhumatologique à Lomé (Togo)
}

\author{
Kakpovi K. \\ Koffi-TessioV \\ Service de Rhumatologie, CHU Sylvanus Olympio, \\ Université de Lomé-Togo,
}

Houzou $\boldsymbol{P}$.

Service de Rhumatologie, CHU Kara, Université de Kara-Togo,

Fianyo $E$.

Service de Rhumatologie, CHR Lomé Commune, Université de Lomé-Togo,

Kolou M.

Service de Laboratoire de biologie moléculaire et d'immunologie de la Faculté des sciences de la santé de l'Université de Lomé (BIOLIM FSS/UL), Lomé -Togo

\section{Kuéviakoé M.I}

Service d'Hématologie, CHU Campus, Université de Lomé-Togo,

\section{Padaro $\boldsymbol{E}$.}

Service d'Hématologie, CHU Sylvanus Olympio, Université de Lomé-Togo,

\section{Tagbor $K$.}

Service de Rhumatologie, Hôpital de Bè, Université de Lomé-Togo,

\section{Oniankitan $\boldsymbol{O}$.}

Service de Rhumatologie, CHR Lomé Commune, Université de Lomé-Togo, Mijiyawa $M$.

Service de Rhumatologie, CHU Sylvanus Olympio,

Université de Lomé-Togo,

Abstract

Objective: To determine epidemiological, clinical, therapeutic aspects and outcomes of rheumatoid arthritis (RA) in rheumatologic consultation at lome (Togo). Patients and method: This was retrospective

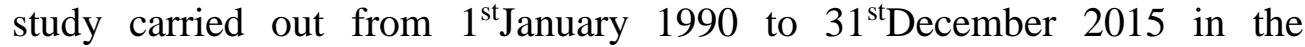
rheumatology department. The study included all patients suffered from RA in rheumatologic consultations and who fulfilled the 2010 ACR and EULAR's criteria. 
Results: Ninety two (77 women and 15 men) out of 25.992 patients $(0.3 \%)$ examined in 25 years had suffered from RA. The mean age at admission was 42 years (range: $17-82$ years). The median duration of the diseases was four years (range: 14days - 20 days). The diseases onset was polyarticular with $86 \%$ of the patients and oligoarticular with the thirteen others $(14 \%)$. The proximal interphalangeal (PIP) joints and metacarpophalangeal (MCP) joints are involved in $81,5 \%$ of cases; and the wrists in $77,2 \%$ of cases. The rheumatoid hip was observed in four patients. Forty-two of the patients $(45,6 \%)$ presented RA deformities. Bilateral MCP and IPP joint early erosion was observed in 36 patients $(39,1 \%)$ and bilateral carpal diffuse osteoporosis in 44 patients $(47,8 \%)$. The ankylosis of the carpal bones was observed in 26 patients $(28,2 \%)$. Rheumatoid factor was positive in $44 \%$ of patients. Methotrexate was the most commonly disease-modifying antirheumatic drugs used in $44,4 \%$ of the patients. The disease was improved in $89 \%$ of patients. Conclusion: Rheumatoid arthritis seems relatively rare in Togo. It's diagnosis is often made at the established phase and methotrexate remains the cornerstone of the treatment.

Keywords: Epidemiology/ Rheumatoid arthritis/ Togo/ Africa

\section{Résumé :}

Objectif : déterminer le profil épidémiologique, clinique, paraclinique, thérapeutique et évolutif de la polyarthrite rhumatoïde (PR) en consultation rhumatologique à Lomé (Togo)

Patients et Méthodes : Il s'agissait d'une étude rétrospective réalisée du $1^{\text {er }}$ Janvier 1990 au 31 décembre 2015 dans le service de rhumatologie. L'étude a inclus tous les patients vus en consultation de rhumatologie pour PR et répondant aux critères de l'ACR et de l'EULAR de 2010.

Résultats : 92 (77 femmes, 15 hommes) des 25992 patients ( $0,3 \%)$ consultés en 25 ans ont souffert de PR. L'âge moyen à l'admission était de 42 ans (extrêmes : 17 ans et 82 ans). La durée moyenne d'évolution était de 4 ans (extrêmes : 14 jours et 20 ans). Le début était polyarticulaire chez 79 patients (86\%) et oligoarticualaire chez les 13 autres (14\%). La topographie des atteintes était dominée par l'atteinte des mains $(81,5 \%)$ et des poignets (77,2\%).Une coxite était observée chez quatre patients. Quarante-deux des patients $(45,6 \%)$ présentaient des déformations caractéristiques de la PR. Une carpite bilatérale a été observée chez 36 patients $(39,1 \%)$ et une déminéralisation en bande chez 44 patients $(47,8 \%)$. La carpite était fusionnante chez 26 patients $(28,2 \%)$. Le Facteur rhumatoïde était positif dans $44,4 \%$ des cas. Le méthotrexate était le traitement de fond le plus utilisé dans $40,2 \%$ des cas.L'évolution a été favorable dans $89 \%$ des cas. 
Conclusion : La polyarthrite rhumatoïde semble relativement rare au Togo, son diagnostic est souvent fait à la phase d'état et le méthotrexate demeure le pilier du traitement.

Mots clés : Epidémiologie/ polyarthrite rhumatoïde/ Togo/ Afrique

\section{Introduction}

La polyarthrite rhumatoïde (PR) est une pathologie inflammatoire très hétérogène à composante auto-immune. Elle est le plus fréquent des rhumatismes inflammatoires chroniques de l'adulte. La prévalence mondiale est estimée entre 0,5 et 1\% (Silman AJ et al, 2002 ; Gabriel SE, 2001). En Afrique, des différences de prévalence sont observées selon la région et selon les zones rurales ou urbaines (Mijiyawa M, 1995 ; Bileckot R et al, 1998). Sa fréquence hospitalière est estimée à 2,18\% au Burkina-Faso (Ouédraogo DD et al, 2011), à $0,8 \%$ au Bénin (Avimadjè $\mathrm{M}$ et al, 2009) et à $0,45 \%$ au Togo (Houzou P et al, 2013). La PR est une affection potentiellement grave car elle peut engager le pronostic fonctionnel de par ses conséquences articulaires et vital de par ses atteintes viscérales. Elle pose souvent et particulièrement en Afrique noire, de difficiles problèmes diagnostiques surtout au stade précoce et des problèmes thérapeutiques. Dans le but d'identifier les patients plus précocement dans le cours naturel du développement de leur maladie, les critères ACR de 1987 ont été révisés en critères ACR/EULAR 2010 (Fautrel $B$ et al, 2010). Notre étude a pour objectif de déterminer le profil épidémiologique, clinique, paraclinique, thérapeutique et évolutif de la polyarthrite rhumatoïde en consultation rhumatologique à Lomé (Togo).

\section{Patients et Méthodes}

Il s'est agi d'une étude rétrospective réalisée du $1^{\text {er }}$ Janvier 1990 au 31 décembre 2015 dans le service de rhumatologie du CHU Sylvanus Olympio. Tous les patients vus en consultation de rhumatologie pour polyarthrite rhumatoïde et répondant aux critères de l'ACR/ l'EULAR de 2010 (Fautrel $B$ et al, 2010) avec un score $\geq 6$ ont été inclus dans l'étude. Les patients présentant une goutte polyarticulaire, une polyarthrite infectieuse ou tout autre rhumatisme inflammatoire chronique, une polyarthrite indifférenciée et/ou insuffisamment explorés avaient été exclus de l'étude. Les données démographiques (âge, sexe), cliniques (durée d'évolution de la maladie, mode de début, évaluation de la douleur par l'échelle visuelle analogique, manifestations articulaires et extra-articulaires), paracliniques, thérapeutiques et évolutives des patients ont été recueillies à partir de leurs dossiers. L'association d'une asthénie, d'une anorexie, d'un amaigrissement (un amaigrissement > 10\% du poids antérieur était considéré comme massif) 
et d'une pâleur clinique ou la présence d'au moins trois de ces symptômes était considérée comme une altération de l'état général. La vitesse de sédimentation supérieure à $20 \mathrm{~mm}$ à la première heure était considérée comme accélérée. La C-Protéine Réactive (CRP) supérieure à 6 mg/l était considérée comme élevée. L'anémie correspondait à une concentration en hémoglobine inférieure à $10 \mathrm{~g} / \mathrm{dl}$. La détermination du facteur rhumatoïde a été faite par le test ELISA et est considéré comme positive pour une valeur supérieure à $10 \mathrm{UI} / \mathrm{ml}$. La détermination des auto- anticoprs Anti- Peptides Cycliques Citrullinés 2 (anti-CCP2) a été faite par ELIA CCP (Thermo Fisher Scientific) et est considérée comme positive pour une valeur supérieure à $10 \mathrm{U} / \mathrm{ml}$. La sérologie rétrovirale a été réalisée chez tous nos patients. Une créatininémie supérieure à $12 \mathrm{mg} / 1$ était considérée comme une insuffisance rénale. La radiographie des mains prenant les poignets et du thorax a été systématiquement réalisée chez tous nos patients. L'évolution était jugée favorable sur la base de l'amendement des signes cliniques tels la douleur, l'altération de l'état général. Les données ont été analysées avec le logiciel Epi Info 3.5.1. Les résultats ont été exprimés en termes de fréquence et de moyennes \pm écart-type. Les tests d'Anova et de Fischer ont été utilisés. Le $\mathrm{p}<0.05$ a été considéré comme significatif.

\section{Résultats}

Quatre-vingt-douze des 25992 patients (0,3\%) consultés en 25 ans ont souffert de Polyarthrite rhumatoïde. Les 92 patients étaient répartis en 77 femmes $(83,7 \%)$ et 15 hommes $(16,3 \%)$ donnant une sex-ratio de 5,1. L'âge moyen à l'admission était de $42 \pm 5,9$ ans avec des extrêmes de 17 ans et 82 ans. Les tranches d'âge de 21 à 31 ans et 32 à 42 ans étaient les plus touchées respectivement dans $25 \%$ des cas et $22,8 \%$ des cas. Seuls dix patients $(10,9 \%)$ avaient un âge supérieur ou égal à 65 ans. Parmi ces 92 patients, $77(83,7 \%)$ habitaient en milieu urbain, contre 15 patients $(16,3 \%)$ en milieu rural. Les antécédents étaient représentés par l'hypertension artérielle $(10,9 \%)$, les avortements $(07,6 \%)$, le diabète $(04,3 \%)$ et la PR familiale $(02,3 \%)$. La durée moyenne d'évolution était de 4 ans avec des extrêmes de 14 jours et de 20 ans.L'installation était progressive dans $89 \%$ des cas et brutale dans $11 \%$ des cas. Le début était polyarticulaire chez 79 patients $(86 \%)$ et oligarticualaire chez les 13 autres (14\%).Chez les 79 patients ayant un début polyarticulaire, l'atteinte était symétrique et bilatérale chez 77 patients $(97,5 \%)$ et bilatérale et asymétrique chez deux patients $(2,5 \%)$. Le siège des atteintes était dominé par les mains $(81,5 \%)$ et les poignets $(77,2 \%)$ (Tableau 1).

Tableau 1 : Répartition des patients selon le siège des atteintes articulaires

\begin{tabular}{ccc}
\multicolumn{2}{c}{ Tableau 1 : Répartition des patients selon le siège des atteintes articulaires } \\
\hline & Nombre & Pourcentage \\
\hline Mains & 75 & 81,5
\end{tabular}




$\begin{array}{ccc}\text { Poignets } & 71 & 77,2 \\ \text { Genoux } & 65 & 70,6 \\ \text { Chevilles } & 59 & 64,1 \\ \text { Epaules } & 47 & 57,6 \\ \text { Pieds } & 46 & 50\end{array}$

Quarante-deux des patients $(45,6 \%)$ présentaient des déformations caractéristiques de la PR (Tableau 2).

Tableau 2 : Répartition des patients en fonction des déformations

\begin{tabular}{ccc}
\hline & Nombre & Pourcentage \\
\hline Coup de vent cubital & 21 & 22,8 \\
Dos de chameau & 12 & 13,0 \\
Doigt en boutonnière & 11 & 12,0 \\
Touche de piano & 07 & 07,6 \\
Doigt en col de cygne & 05 & 05,5 \\
Pouce en Z & 04 & 04,3 \\
Doigt en maillet & 02 & 02,2 \\
\hline
\end{tabular}

Les manifestations extra-articulaires ( 38 cas ; 41,30\%) étaient hématologique $(27,2 \%)$, pulmonaire (pneumopathie interstitielle: 06,5\%), rénale (insuffisance rénale aiguë : 02,2\%), cutanée (nodule rhumatoïde : 02,2\%), neurologique (neuropathie périphérique : $02,2 \%$ ) et cardiaque (péricardite : $01,1 \%$ ). La vitesse moyenne de sédimentation était à $71,8 \pm 38,1 \mathrm{~mm}$ à la 1 ère heure avec des extrêmes de $02 \mathrm{~mm}$ et $150 \mathrm{~mm}$; elle était accélérée chez 82 patients (89\%). La CRP était positive chez 80 patients (87\%).Le taux d'hémoglobine moyen était à $12,1 \pm 1,5 \mathrm{~g} / \mathrm{dl}$ avec des extrêmes de $4,8 \mathrm{~g} / \mathrm{dl}$ et $16,6 \mathrm{~g} / \mathrm{dl}$. Vingt-trois patients $(25 \%)$ avaient une anémie. Le facteur rhumatoïde a été réalisé chez neuf patients et était positif dans $44,4 \%$ des cas. Les anticorps anti-peptides cycliques citrullinés étaient positifs chez cinq patients $(55,5 \%)$. Une coxite était observée chez quatre patients $(4,3 \%)$. La radiographie des mains a mis en évidence une carpite bilatérale chez 36 patients $(39,1 \%)$ et une déminéralisation en bande chez 44 patients $(47,8 \%)$. La carpite était fusionnante chez 26 patients $(28,2 \%)$. Le méthotrexate était le traitement de fond le plus utilisé dans $40,2 \%$ des cas (Tableau 3 ).

Tableau 3 : Répartition des patients en fonction des traitements de fond utilisés

\begin{tabular}{ccc}
\hline & Nombre & Pourcentage \\
\hline Méthotrexate & 37 & 40,2 \\
Hydroxychloroquine & 11 & 12,0 \\
Sulfasalazine & 05 & 05,4 \\
Léflunomide & 01 & 01,1 \\
\hline
\end{tabular}


Dans 48,9\% des cas, aucun traitement de fond n'a été utilisé. L'évolution a été favorable dans $89 \%$ des cas et défavorable dans $11 \%$. Deux cas $(2,2 \%)$ de décès ont été observés.

\section{Discussion}

La fréquence de la PR au CHU SO est de 0,3\%. Cette faible fréquence est conforme aux données de la littérature en Occident (Guillemin F et al, 2005 ; Alamanos Y et al, 2006), et en Afrique (Ouédraogo DD et al, 2011 ; Avimadjè M, 2009). La prédominance féminine ( 5 femmes pour un homme) observée dans notre étude a été décrite par plusieurs auteurs (Ouédraogo DD et al, 2011 ; Malemba JJ et al, 2012). L'âge moyen de nos patients correspond à l'âge moyen de prédilection de la maladie qui est située entre 40 et 60 ans (Malemba JJ et al, 2012; Atouf O, 2008). La durée d'évolution longue (48 mois) et l'installation progressive $(89,1 \%)$ observées dans notre série expliquent la fréquence élevée des déformations. les mêmes constatations ont été fait par Ouedraogo et al (2011) au Burkina-Faso et par Eti et al (2000) en Côte d'Ivoire. Ce long retard de consultation peut également s'expliquer par le faible développement de la rhumatologie en milieu africain, la méconnaissance de la pathologie par les médecins généralistes et surtout par le recours aux tradipraticiens. Dans notre série, la principale déformation était le coup de vent cubital tout comme dans les autres séries africaines (Avimadjè $\mathrm{M}$ et al, 2009 ; Eti E et al, 2000).Comme décrite par plusieurs auteurs (Bileckot R et al, 1998 ; Atouf O et al, 2008 ; Eti E, 2000), la PR a un début polyarticulaire tout comme dans notre étude. La topographie des atteintes est dominée dans notre étude par l'atteinte des inter-phalangiennes proximales des mains. Le même constat a été fait par Bileckot et al (1998) au Congo. Ce résultat est contraire à ceux trouvés par Ouedraogo et al (2011) au Burkina-Faso et par Avimadjè et al (2009) au Bénin, où il y avait une prédominance des poignets. La prédominance des atteintes aux articulations de la main et du poignet classiquement décrite dans la littérature est respectée dans notre série. La symétrie et la bilatéralité des atteintes observées dans 97,5\% des cas est conforme avec les données de la littérature (Ouédraogo DD et al, 2011; Eti E et al, 2000). Les manifestations extra articulaires étaient fréquentes dans notre série $(41,30 \%)$ contrairement aux séries ivoiriennes et sénégalaises (Mijiyawa M, 1995). La rareté de nodules rhumatoïdes sous cutanés en Afrique de l'Ouest rapportées dans les séries nigérianes 4 cas sur 71 (Mijiyawa $M$ et al, 1995), et sénégalaise $1,06 \%$ (Roux H, 2002) est confirmée dans notre série. Ils sont plutôt fréquents en Afrique du Sud 25 \% (Mijiyawa M, 1995). La présence de nodules sous cutanés est associée aux formes sévères de PR, et sa rareté dans la sous-région plaiderait pour une bénignité apparente de la PR en Afrique Occidentale (Roux H, 2002).Le syndrome sec était absent dans notre 
série contrairement aux différentes proportions retrouvées au Sénégal (60,5\%) (Roux H, 2002) et au Burkina-Faso (31\%) (Ouédraogo DD et al, 2011). Le syndrome sec peut apparaître tardivement au cours de l'évolution de la PR. Dans notre série, la PR était séropositive dans 44,4\% et les antiCCP étaient positifs dans $55,5 \%$ des cas.Ces taux sont inférieurs à ceux trouvés en Afrique (Ouédraogo DD et al, 2011 ; Singwe-Ngandeu M et al, 2010 ; Ntsiba H et al, 2009) et en Occident (Sany J, 2003). Ceci s'explique par le fait que ces analyses ne se font pas à Lomé, que le coût (30.000 FCFA $=$ SMIG togolais) ne soit pas à la portée de nos patients et que le diagnostic était clinique dans la majorité des cas. Le traitement symptomatique a été dominé dans notre série par 1'utilisation des AINS et la corticothérapie. Cette attitude thérapeutique a été adoptée chez la plupart des patients dans les séries africaines (Ouédraogo DD et al, 2011 ; Avimadjè M et al, 2009). Le méthotrexate demeure le traitement de fond le plus utilisé en raison de son accessibilité et de son innocuité.

\section{Conclusion}

La polyarthrite rhumatoïde semble relativement rare au Togo, son diagnostic est souvent fait à la phase d'état et le méthotrexate demeure le pilier du traitement.

\section{References:}

1. Silman,AJ., Pearson, JE (2002). Epidemiology and genetics of rheumatoidarthritis. ArthritisRes;4(Suppl 3):S265 - 72.

2. Gabriel, SE (2001). The epidemiology of rheumatoidarthritis. Rheum Dis Clin North Am;27:269- 281.

3. Mijiyawa, M(1995). Aspects épidémiologiques et sémiologiques de la polyarthrite rhumatoïde dans le Tiers- monde. Rev Rhum ; 62 : 127 $-132$.

4. Bileckot, R., Malonga, AC (1998). La polyarthrite rhumatoïde en milieu hospitalier au Congo Brazaville à propos de 36 cas. Rev Rhum ; 65 : 333-7.

5. Ouédraogo, DD., Singbo, J., Diallo, O., Sawadogo, SA. Tieno, H., Drabo, YJ (2011).Rheumatoid arthritis in Burkina Faso: clinical and serological profiles. Clin Rheumatol ; 30:1617-21.

6. Avimadjè, M., Gounongbé, M., Zomalhèto, Z (2009). La polyarthrite rhumatoïde au

7. CNHU de Cotonou. Le Bénin Médical; 57 (42/43): 55-7.

8. Houzou, P., Oniankitan, O., Kakpovi, K., Koffi-Tessio, VES., Tagbor, KC., Fianyo, E., Mijiyawa, M (2013). Profil des affections rhumatismales chez 13517 patients ouest-africains. Tunis Méd; 91 (1) : 16-20. 
9. Fautrel, B., Rincheval, N., Combe, B (2010). Degré de concordances des critères de classification de l'ACR 1987 et l'ACR/EULAR 2010 : une analyse à partir des données de la cohorte ESPOIR. Rev Rhum ; 77 (suppl3) : 235-6.

10. Guillemin, F., Saraux, A., Guggenbuhl, P. et al (2005). Prevalence of Rheumatoidarthritis in France: 2001. Ann Rheum Dis.; 64:1427-30.

11. Alamanos, Y., Voulgari, PV., Drosos, AA. et al (2006). Incidence and prevalence of rheumatoid arthritis based on the 1987 American College of Rheumatology criteria: asystematic review. Arthritis Rheum; 36:182-188.

12. Malemba, JJ., Mbuyi-Muamba, JM., Mukaya, J., Bossuyt, X., Verschueren, P., Westhovens, R (2012). The epidemiology of rheumatoid arthritis in Kinshasa, DemocraticRepublic of Congo.a population-based study. Rheumatology; 51: 1644 - 1647.

13. Atouf, O., Benbouazza, K., Brick, C., Bzami, F., Bennani, N., HajjajHassouni, N. et al (2008). Polymorphisme HLA et polyarthrite rhumatoïde précoce dans la population marocaine. Rev. Rhum ; 75 : $824-30$.

14. Eti, E., Sylla, M., Daboiko, JC. et al (2000). Contribution of the study of the rheumatoid arthritis in IvorianHospitals. About 90 cases. Rev Int Sciences Méd ; 2 : 71-8.

15. Mijiyawa M (1995). Aspects sémiologiques et épidémiologiques des maladies rhumatismales en Afrique Noire. Sem Hôp Paris ; 71 : 91223.

16. Roux H (2002).Polyarthrite rhumatoïde en Afrique subsaharienne. Rev Rhum; 69: 797-800.

17. Singwe-Ngandeu, M., Finckh, A., Bas, S., Tiercy, JM., Gabay, C (2010).. Diagnosis value of anti-cycliccitrullinated peptides and rheumatoid arthritis patients. Arth ResTher ; 12 : 1-7.

18. Ntsiba, H., Ngandeu-Singwe, M., Mbuyi-Muamba, JM (2009). Rheumatoidarthritis in central Africa. Epidemiological and clinical aspects [abstract]. Rev Rhum ; 76:1184-1185.

19. Sany J (2003). Rheumatoid arthritis of the adult Current Design. Paris:John Libbey Eurotext: 5-7. 